A N N A LES

UNIVERSITATIS MARIAE CURIE-SKŁODOWSKA

L UBLIN - P OLONIA

VOL. LXIX, NO. 1, 2015

SECTIO A

$1-4$

EDOARDO BALLICO

\title{
Components with the expected codimension in the moduli scheme of stable spin curves
}

\begin{abstract}
Here we study the Brill-Noether theory of "extremal" Cornalba's theta-characteristics on stable curves $C$ of genus $g$, where "extremal" means that they are line bundles on a quasi-stable model of $C$ with $\sharp(\operatorname{Sing}(C))$ exceptional components.
\end{abstract}

1. Introduction. For any integer $g \geq 2$ let $\bar{M}_{g}$ denote the moduli space of stable curves of genus $g$ over an algebraically closed field $\mathbb{K}$ such that $\operatorname{char}(\mathbb{K})=0$. Fix any $Y \in \bar{M}_{g}$. The topological type (if $\mathbb{K}=\mathbb{C}$ ) or the equisingular type (for arbitrary $\mathbb{K}$ ) $\tau$ may be described in the following way. Fix an ordering $Y_{1}, \ldots, Y_{s}$ of the irreducible components of $Y$. The type $\tau$ is uniquely determined by the string of integers listing the geometric genera of $Y_{1}, \ldots, Y_{s}$, the integers $\sharp\left(\operatorname{Sing}\left(Y_{i}\right)\right), 1 \leq i \leq s$, and the integers $\sharp\left(Y_{i} \cap Y_{j}\right)$, $1 \leq i<j \leq s$ (see [1], p. 99). Recently, the Brill-Noether theory of thetacharacteristics of smooth curves had a big advances due to a solution by L. Benzo ([3]) of a conjecture of G. Farkas ([6], Conjecture 3.4). In this note we show that such a result may be used for the study of the Brill-Noether theory of Cornalba's theta-characteristics on $\bar{M}_{g}$. Indeed, we will check that for the extremal theta-characteristics we are looking for in this note the existence of such a theta-characteristic on $Y$ with prescribed number of

2010 Mathematics Subject Classification. 14H10; 14H51; 14H42.

Key words and phrases. Stable curve, theta-characteristic, spin curve, Brill-Noether theory.

The author was partially supported by MIUR and GNSAGA of INdAM (Italy). 
linearly independent sections, $r+1$, is equivalent to the existence of thetacharacteristics $E_{1}, \ldots, E_{s}$ on the normalizations $C_{1}, \ldots, C_{s}$ of $Y_{1}, \ldots, Y_{s}$ and with $\sum_{i=1}^{s} h^{0}\left(C_{i}, A_{i}\right)=r+1$.

Let $\mathcal{S}_{g}, g \geq 2$, be the set of all theta-characteristics on smooth genus $g$ curves, i.e. the set of all pairs $(C, L)$ with $C \in M_{g}, L \in \operatorname{Pic}(C)$ and $L^{\otimes 2} \cong \omega_{C}$. For all integers $r \geq-1$ set $\mathcal{S}_{g}^{r}:=\left\{(C, L) \in \mathcal{S}_{g}: h^{0}(L)=\right.$ $r+1\}$. The set $\mathcal{S}_{g}^{r}$ is a locally closed subset of $\mathcal{S}_{g}$ and each point of it has codimension at most $\left(\begin{array}{c}r+1 \\ 2\end{array}\right)$ in $\mathcal{S}_{g}$ ([8], part (ii) of Theorem 1.10). Maurizio Cornalba proved the existence of a compactification $\overline{\mathcal{S}}_{g}$ of $\mathcal{S}_{g}$ equipped with a finite morphism $u_{g}: \overline{\mathcal{S}}_{g} \rightarrow \bar{M}_{g}$ such that each fiber of $u_{g}$ has cardinality $2^{2 g}$ ([5], Proposition 5.2 and first part of $\left.\S 3\right)$. There are many topological types for which the Brill-Noether theory of theta-characteristics with $r+1$ linearly independent sections never occurs in the expected codimension, i.e. in codimension $\left(\begin{array}{c}r+1 \\ 2\end{array}\right)$ (see [2] for a description of all theta-characteristics with $g$ linearly independent sections). The claim of this note is that to study the Brill-Noether theorem of $\overline{\mathcal{S}}_{g} \backslash \mathcal{S}_{g}$ one needs to distinguish the quasi-stable model on which a Cornalba's theta-characteristic lives as a line bundle. In other compactifications of $\mathcal{S}_{g}$ (as in [9]) torsion-free sheaves are used; prescribing the non-locally free points of these sheaves on some $C \in \bar{M}_{g}$ is equivalent to prescribe the images in $\operatorname{Sing}(C)$ of the quasistable model of $C$ on which a Cornalba's theta-characteristic "is" a line bundle (it is not quite a line bundle $L$, but a line bundle up-to inessential isomorphisms and we also need to prescribe the line bundle $L^{\otimes 2}$ ([5], Lemma 2.1 and first part of §3)). None of these problems affect the Brill-Noether theory for the theta-characteristics we will consider in this note (we call them the maximally singular ones). For these theta-characteristics the computation of $h^{0}$ is reduced to the computations of $h^{0}$ for theta-characteristics on the normalizations of all the irreducible components of the given $C \in \bar{M}_{g}$. Hence the existence part is reduced to an existence part on smooth curves for all genera up to $g$. There is a natural injective morphism from $\overline{\mathcal{S}}_{g}$ into Caporaso's compactification $\bar{P}_{g-1, g}([4])$ of the set of all degree $g-1$ line bundles on $M_{g}([7])$. A Cornalba's theta-characteristic associated to a stable curve $C$ is said to be maximally singular if it is a line bundle on the quasi-stable model $C^{\prime}$ of $C$ obtained blowing up all singular points of $C$. A Cornalba's theta-characteristic on $C$ is maximally singular if and only if it induces a theta-characteristic on the normalization of $C$ ([5], Lemma 1.1). If $C$ has compact type, then each theta-characteristic on $C$ is maximally singular, because for each $S \subset \operatorname{Sing}(C)$, the quasi-projective curve $C \backslash S$ has $\sharp(S)+1$ connected components.

Obviously $\left(\begin{array}{l}a \\ 2\end{array}\right)=0$ for $a=0,1$. Define the function $\alpha: \mathbb{N} \rightarrow \mathbb{N}$ in the following way. Set $\alpha(0):=1$ and $\alpha(1):=1$. For all integers $q \geq 2$ let $\alpha(q)$ 
be the maximal positive integer such that $\left(\begin{array}{c}\alpha(q)+1 \\ 2\end{array}\right) \leq q$. We have $\alpha(2)=1$ and $\alpha(3)=2$.

Theorem 1. Fix a type $\tau$ for genus $g$ stable curves. Let $q_{1}, \ldots, q_{s}$ be the geometric genera of the irreducible components of stable curves with type $\tau$. Fix integers $a_{i}, 1 \leq i \leq s$, such that $0 \leq a_{i} \leq \alpha\left(q_{i}\right)$ for all $i$ and set $r:=-1+\sum_{i=1}^{s} a_{i}$. Then there is an irreducible component $\Gamma$ of the set of all maximally singular Cornalba's theta-characteristics for stable curves with type $\tau$ with codimension $\sum_{i=1}^{s}\left(\begin{array}{c}a_{i} \\ 2\end{array}\right)$ and such that for a general $(Y, L) \in \Gamma$ with $Y=Y_{1} \cup \cdots \cup Y_{s}$, each $Y_{i}$ of geometric genus $q_{i}$ and $h^{0}\left(C_{i}, L \mid C_{i}\right)=a_{i}$ for all $i$, where $C_{i}$ is the normalization of $Y_{i}$.

In most cases no component satisfying the thesis of Theorem 1 may be smoothable, i.e., it is in the closure inside $\overline{\mathcal{S}}_{g}$ of an irreducible component of $\mathcal{S}_{g}^{r}$, just because $r$ may be very high.

\section{The proof.}

Remark 1. Fix an integer $q \geq 0$ and a smooth genus $q$ curve $D$. If $q \geq 3$, then assume that $D$ is general in its moduli space. A corollary of GiesekerPetri theorem (case $q \geq 3$ ) ([1], Proposition 21.6.7) or Riemann-Roch gives that every theta-characteristic $A$ on $D$ satisfies $h^{0}(D, A) \leq 1$. We will only use the existence of theta-characteristics $A, B$ on $D$ such that $h^{0}(D, A)=0$ and $h^{0}(D, B)=1$.

Remark 2. Notice that $\mathcal{S}_{3}^{1}$ has codimension 1 in $M_{3}$, because the hyperelliptic locus of $M_{3}$ has dimension 5. By [6], Theorem 1.2, $\mathcal{S}_{g}^{1}$ has a component of the expected codimension, 1 , for all $g \geq 3$.

Lemma 1. Let $Y$ be a reduced projective curve such that $Y=C \cup T$ such that $T \cong \mathbb{P}^{1}, \sharp(C \cap T)=2$ and each point of $C \cap T$ is a nodal point of $Y$. Let $R$ be any line bundle on $Y$ such that $\operatorname{deg}(R \mid T)=1$. Then $h^{i}(Y, R)=h^{i}(C, R \mid C)$, $i=0,1$.

Proof. We have the Mayer-Vietoris exact sequence:

$$
0 \rightarrow R \rightarrow R|C \oplus R| T \rightarrow R \mid C \cap T \rightarrow 0
$$

Since $\operatorname{deg}(C \cap T)=2, \operatorname{deg}(R \mid T)=1$ and $R$ is a line bundle, the restriction map $H^{0}(T, R \mid T) \rightarrow H^{0}(C \cap T, R \mid C \cap T)$ is an isomorphism. Hence (1) gives $h^{i}(Y, R)=h^{i}(C, R \mid C), i=0,1$.

Proof of Theorem 1. Fix a stable curve $Y=Y_{1} \cup \cdots \cup Y_{s}$ with each $Y_{i}$ of geometric genus $q_{i}$. Let $C=C_{1} \sqcup \cdots \sqcup C_{s}$ be the normalization of $Y$ with $C_{i}$ the normalization of $Y_{i}$. Assume for the moment the existence of a theta-characteristic $A_{i}$ on $C_{i}$ such that $h^{0}\left(C_{i}, A_{i}\right)=a_{i}$ and let $A^{\prime}$ be the line bundle on $C_{1} \sqcup \cdots \sqcup C_{s}$ with $A^{\prime} \mid C_{i}=A_{i}$ for all $i$. Let $Y^{\prime}$ be the quasistable curve with $Y$ as its stable reduction and with $\sharp(\operatorname{Sing}(Y))$ exceptional components. Let $A$ be any line bundle on $Y^{\prime}$ with $A^{\prime}$ as its pull-back to 
$C$ and $\operatorname{deg}(A \mid J)=1$ for each exceptional component $J$ of $Y^{\prime}$. Applying $\sharp(\operatorname{Sing}(Y))$ times Lemma 1 , we get $h^{0}\left(Y^{\prime}, A\right)=r+1$. $A$ is a totally singular Cornalba's theta-characteristic. Now we count the parameters. By the definitions of the integers $\alpha\left(q_{i}\right)$ and $a_{i}$ we have $q_{i} \geq\left(\begin{array}{c}a_{i}+1 \\ 2\end{array}\right)$ for all $i$ if $a_{i} \geq 2$. By [3], Theorem 1.2, there is an irreducible component $\Gamma_{i} \subset \mathcal{S}_{q_{i}}^{a_{i}-1}$ if $a_{i} \geq 2$. For the case $a_{i}=0$ use Remark 1. For the case $a_{i}=1$ use Remark 2. Taking all $(Y, A)$ coming from all $\left(C_{i}, A_{i}\right) \in \Gamma_{i}$, we get a family of curves $Y$ with codimension $\sum_{i=1}^{s}\left(\begin{array}{c}a_{i} \\ 2\end{array}\right)$ in the subset $M(\tau) \subset \bar{M}_{g}$ with type $\tau$. This is a maximal family (i.e. an open subset of an irreducible component of $\overline{\mathcal{S}}_{g}^{r}$ ), because each $\Gamma_{i}$ is a maximal family and for all $Y \in M(\tau)$ the fiber $u_{g}^{-1}(Y)$ has the same number of elements.

\section{REFERENCES}

[1] Arbarello, E., Cornalba, M., Griffiths, P. A., Geometry of Algebraic Curves. Vol. II, Springer, Berlin, 2011.

[2] Ballico, E., Sections of theta-characteristics on stable curves, Int. J. Pure Appl. Math. 54, No. 3 (2009), 335-340.

[3] Benzo, L., Components of moduli spaces of spin curves with the expected codimension, Mathematische Annalen (2015), DOI 10.1007/s00208-015-1171-6, arXiv:1307.6954.

[4] Caporaso, L., A compactification of the universal Picard variety over the moduli space of stable curves, J. Amer. Math. Soc. 7, No. 3 (1994), 589-660.

[5] Cornalba, M., Moduli of curves and theta-characteristics. Lectures on Riemann surfaces (Trieste, 1987), World Sci. Publ., Teaneck, NJ, 1989, 560-589.

[6] Farkas, G., Gaussian maps, Gieseker-Petri loci and large theta-characteristics, J. Reine Angew. Math. 581 (2005), 151-173.

[7] Fontanari, C., On the geometry of moduli of curves and line bundles, Atti Accad. Naz. Lincei Cl. Sci. Fis. Mat. Natur. Rend. Lincei (9) Mat. Appl. 16, No. 1 (2005), 45-59.

[8] Harris, J., Theta-characteristics on algebraic curves, Trans. Amer. Math. Soc. 271 (1982), 611-638.

[9] Jarvis, T. J., Torsion-free sheaves and moduli of generalized spin curves, Compositio Math. 110, No. 3 (1998), 291-333.

Edoardo Ballico

Department of Mathematics

University of Trento

38123 Povo (TN)

Italy

e-mail: ballico@science.unitn.it

Received August 15, 2013 